\title{
Comparative Performance Analysis of AODV and AODV-MIMC Routing Protocols for Mobile Ad hoc Networks
}

\author{
P.Periyasamy \\ Department of Computer Science and Applications, Sree Saraswathi Thyagaraja College, Pollachi - 642 107, Tamil \\ Nadu, India. \\ Email: pereee@yahoo.com \\ Dr.E.Karthikeyan \\ Department of Computer Science, Government Arts College, Udumalpet - 642 126, Tamil Nadu, India. \\ Email: e_karthi@yahoo.com
}

\begin{abstract}
Bandwidth Scarcity is a major drawback in multi-hop ad hoc networks. When a single-interface single-channel (SISC) approach is used for both incoming and outgoing traffic the bandwidth contention between nodes along the path has occurred as well as throughput is degraded. This drawback is overwhelmed by using MIMC approach as well as some of the Quality of Service (QoS) requirements has been enhanced. In this paper we applied multi-interface muti-channel approach to AODV routing protocol, called AODV-MIMC routing protocol and its performance is compared with AODV routing protocol. The simulation results show the Network Life Time, Throughput, and Packet Delivery Ratio of AODV-MIMC routing protocol has been tremendously improved than the AODV routing protocol.
\end{abstract}

Index Terms - SISC, MIMC, AODV-MIMC, Bandwidth Scarcity, Network Life Time, Throughput, and Packet Delivery Ratio.

\section{INTRODUCTION}

In this modern world, Wireless Communication has become indispensible part of life. Research focuses on Mobile Ad hoc NETworks (MANETs), which is a collection of mobile devices by wireless links forming a dynamic topology without much physical network infrastructure such as routers, servers, access points/cables or centralized administration. Each mobile device functions as router as well as node. The main characteristics of MANET are i) Dynamic topologies ii) Bandwidth-constrained links iii) Energy constrained operation and iv) Limited physical security $[1,2]$.

Most of the routing protocols in MANETs have been designed using a single-interface single-channel (SISC) approach. In this approach, a single-interface and singlechannel is commonly used for both incoming and outgoing traffic between nodes along the path. This leads the bandwidth contention and throughput degradation issues. These issues can be tackled by using multi- interface multi-channel (MIMC) approach. The following are the major advantages of MIMC [3, 4] approach:

(i) Capacity Enhancement: Sending and Receiving of data packets by the forwarding nodes at the same time.

(ii) Load Sharing: In order to increase robustness and lower latency, the traffic flow is distributed among the available connections.

(iii) Channel Failure Recovery: Channel errors are possibly avoided because of multiple interfaces and multiple channels.

In this paper, we applied multi-interface multi-channel (MIMC) approach to Ad hoc On-demand Distance Vector (AODV) routing, called AODV-MIMC routing protocol and its performance is compared with AODV routing protocol in terms of Quality of Service (QoS) requirements.

The rest of the paper is organized as follows: Section II briefly discusses the related work. In Section III, we have presented multi-interface and multi-channel approach to AODV routing protocol, called AODV-MIMC routing protocol. In Section IV, the QoS metrics are given. In Section $\mathrm{V}$, the simulation and experimental results are discussed. In Section VI, the conclusions and future work are given.

\section{RELATED WORK}

Many researchers have proposed many different approaches to MAC for utilizing multi-channel and multi-interface in mobile ad hoc networks. In [5] the authors proposed a centralized channel assignment scheme where traffic is directed towards specific gateway nodes in static networks. A hybrid channel assignment scheme [6] assigns some radios statically to a channel and some are dynamically changed their frequencies in the channel. A new channel assignment scheme [7] for utilizing multi-channels that can be reduced channel conflicts by removing hidden channel problem [8].

All the protocols in NS 2.34 have only Single- 
Interface Single-Channel (SISC) support because IEEE $802.11 \mathrm{a} / \mathrm{b} / \mathrm{g}$ requires some modifications on MAC and link layer protocols in order to utilize multi-interface multi-channel (MIMC). In the MIMC [6, 9, 10] approach, the following are the designs of routing interface and channel assignments:

1. A solution for multi-interface which is exploiting multiple channels can be implemented on existing IEEE 802.11 hardware.

2. An interface assignment strategy using interface switching techniques which simplifies the coordination among nodes through the utilization of multiple available channels.

3. A Multiple-Channel Routing (MCR) scheme selects the routes with the highest throughput by accounting the cost of channel diversity and interface switching.

The modifications on MAC and link layer protocols done using P. Kyasanur's and N. H. Vaidya's interface assignment scheme [6] because this scheme is more flexible and versatile among other schemes. Implementation of this scheme on MAC (Medium Access Control) and link layer protocols is carried out using the Technical Report [3].

In [11], the optimal channel assignment and routing problem in wireless mesh networks is overwhelmed. In a distributed algorithm [12], when a node has the number of available channels less than twice the number of network interfaces. In such case, channels are randomly assigned to network interfaces as there is a guarantee that a common channel can be found between any pair of nodes through the pigeonhole principle, otherwise, Skeleton Assisted Channel Assignment Scheme is used.

We propose several modifications on AODV routing protocol to utilize the multi-interface multi-channel (MIMC) scheme efficiently, called AODV-MIMC routing protocol to improve network performance.

\section{AODV-MIMC ROUTING PROTOCOL}

\section{A. AODV (Base Protocol)}

The AODV [13] protocol is a simple and widely used on-demand unipath routing protocol that starts a route discovery process through a route request (RREQ) to the destination throughout the network when needed for MANET. Once a non-duplicate RREQ is received, the intermediate node records the previous hop and checks for a valid and fresh route entry to the destination. The node sends a route reply (RREP) along with a unique sequence number to the source.

On updating the route information, it propagates the route reply and gets additional RREPs if a RREP has either a larger destination sequence number (fresher) or a shorter route found.

\section{B. Multi-Interface Multi-Channel extension to AOMDV protocol}

Table 1 and Table 2 shows the route list structure of
AODV and AODV-MIMC routing protocols respectively. Using [3, 10] AODV-MIMC protocol has developed by extending the AODV routing protocol. AODV-MIMC routing protocol uses channel assignment and interface switching strategies in order to utilize multiple channels and multiple interfaces.

Table 1: Route List Structure of AODV protocol

\begin{tabular}{|l|l|l|l|}
\hline hop_count1 & next_hop1 & last_hop1 & expiration_timeout1 \\
\hline hop_count2 & next_hop2 & last_hop2 & expiration_timeout2 \\
\hline
\end{tabular}

Table 2: Route List Structure of AODV-MIMC protocol

\begin{tabular}{|l|l|l|l|l|}
\hline hop_count1 & next_hop1 & last_hop1 & expiration_timeout1 & interface1 \\
\hline hop_count2 & next_hop2 & last_hop2 & expiration_timeout2 & Interface2 \\
\hline
\end{tabular}

If the Interface Queue associates with K channels and $\mathrm{M}$ interfaces, only one interface is fixed and the remaining interfaces are switchable as shown in Fig. 1.

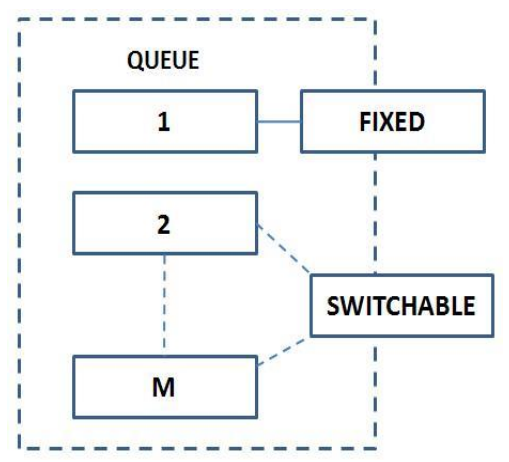

Fig 1: Illustration of the interface queue associated with $\mathrm{K}$ Channels and $\mathrm{M}$ interfaces.

The functions of AODV such as command(), handle(), recvRequest(), forward(), sendRequest(), sendReply(), sendError(), sendHello(), and forwardReply() have modified to adopt with MIMC in order to improve the throughput and network's lifetime. Each node in AODV-MIMC uses both fixed and switchable interfaces and multiple channels. In other words, each node uses one fixed interface and the remaining are switchable interfaces as shown in Fig. 2.

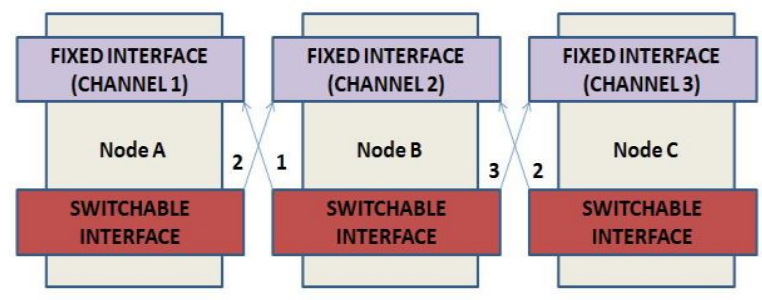

Fig 2: Example of AOMDV-MIMC protocol interface switching operation with $\mathrm{K}=3$ Channels and $\mathrm{M}=2$ interfaces.

\section{QUALITY OF SERVICE (QOS) METRICS}

Quality of Service (QoS) Metrics [14,15] is quantitative measures that can be used to evaluate any ad hoc routing protocol. The following metrics are 
considered in order to compare the performance of ondemand unipath routing protocols AODV and AODVMIMC respectively in terms of variation in speed under Random Waypoint (RWM) in CBR Traffic. The number of bits transferred per second through the traffic medium is called 'network load and the time taken by a node to choose the destination for packet delivery is called 'pause time'.

\section{A. Packet Delivery Ratio}

Packet Delivery Ratio [14, 15] is the ratio of data packets delivered to the destination to those generated by the sources and is calculated as follows:

$$
\text { Packet Delivery Ratio }=\frac{\text { Number of PacketReceived }}{\text { Number of Packets Sent }} \chi 100
$$

\section{B. Throughput}

Throughput $[14,15]$ is the number of bytes received successfully and is calculated by

$$
\text { Throughput }=\frac{\text { Number of bytes received } \chi 8}{\text { Simulationtime } \chi 1000} k b p s
$$

\section{Routing Overhead}

Routing overhead [14,15] is the total number of control packets or routing packets generated by routing protocol during simulation and is obtained by

$$
\text { Routing Overhead }=\text { Number of RTR packets. }
$$

\section{Normalized Routing Overhead}

Normalized Routing Overhead $[14,15]$ is the number of routing packets transmitted per data packet towards destination and calculated as follows:

$$
\text { Normalized Routing Overhead }=\frac{\text { Number of Routing Packets }}{\text { Number of PacketsReceived }}
$$

\section{E. Average End-to-End Delay}

Average End-to-End [14,15] delay is the average time of the data packet to be successfully transmitted across a MANET from source to destination. It includes all possible delays such as buffering during the route discovery latency, queuing at the interface queue, retransmission delay at the MAC, the propagation and the transfer time. The average e2e delay is computed by,

$$
D=\frac{\sum_{i=1}^{n}\left(R_{i}-S_{i}\right)}{n} m \mathrm{sec}
$$

Where $\mathrm{D}$ is the average end-to-end delay, $\mathrm{n}$ is the number of data packets successfully transmitted over the MANET, ' $\mathrm{i}$ ' is the unique packet identifier, $R_{i}$ is the time at which a packet with unique identifier ' $i$ ' is received and $S_{i}$ is the time at which a packet with unique identifier ' $i$ ' is sent. The Average End-to-End Delay should be less for high performance.

\section{F. Packet Loss Ratio}

Packet Loss Ratio $[14,15,16]$ is the ratio between the number of data packets sent and the number of data packets received. It is calculated as follows:

$$
\text { Packet Loss Ratio }=\frac{\text { Number data PacketSent }- \text { Number of data Packets Re ceived }}{\text { Number of Packets Sent }} \chi 100
$$

\section{G. Network Lifetime}

Network Lifetime [16] is defined as the duration from the beginning of the simulation to the first time a node runs out of energy.

\section{H. Number of nodes dying}

The number of nodes [16] died during the simulation time. This can be used to compare how fast the network loses mobile nodes due to battery outage.

\section{SIMULATION AND EXPERIMENT}

\section{A. Simulation Model}

The performance comparison of AODV and AODVMIMC routing protocols are evaluated using NS 2.34 $[18,19,20,21]$. The following Fig 3, illustrates the simulation model and the simulation parameters are described in Table 3.

The result of simulation is generated as trace files and the awk \& perl scripts are prepared to analyze the trace files and produces reports.

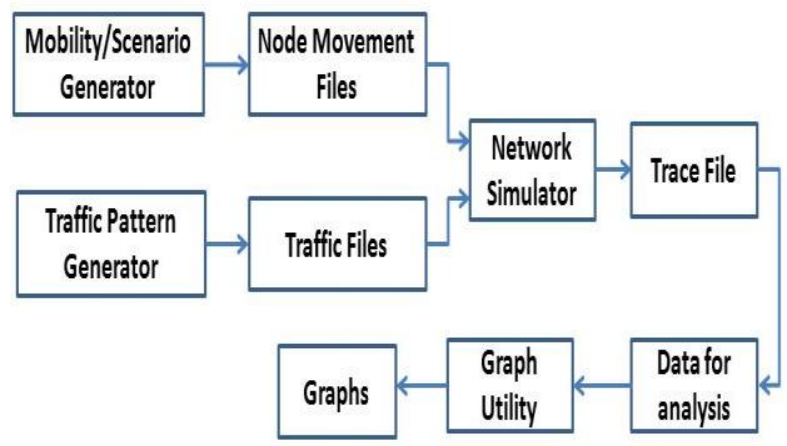

Fig 3: Overview of the simulation model. 
Table 3: Simulation Parameters

\begin{tabular}{|c|c|}
\hline Parameter & Value \\
\hline Simulator & NS-2.34 \\
\hline MAC Type & 802.11 \\
\hline Simulation Time & 100 seconds \\
\hline Channel Type & Wireless Channel \\
\hline Routing Protocols & $\begin{array}{l}\text { AODV } \\
\text { ( } 1 \text { channel and } 1 \text { interface) } \\
\& \text { AODV-MIMC } \\
\text { ( } 3 \text { channels and } 2 \text { interfaces })\end{array}$ \\
\hline Antenna Model & Omni \\
\hline Simulation Area & $2200 \mathrm{~m} \times 600 \mathrm{~m}$ \\
\hline Traffic Type & CBR(udp) \\
\hline Data Payload & 512 bytes/packet \\
\hline Network Loads & 4 packets/sec \\
\hline Number of Connections & 40 \\
\hline Radio Propagation Model & TwoRayGround \\
\hline Idle Power & 0.005 \\
\hline Transmission Power & 12.7 \\
\hline Receiving Power & 12.7 \\
\hline Sleep Power & 0.0001 \\
\hline Transition Power & 0.002 \\
\hline Transition Time & 0.005 \\
\hline Initial Energy & 100 Joules \\
\hline Interface Queue Length & 50 \\
\hline Interface Queue Type & DropTail/PriQueue \\
\hline Number of nodes & 100 \\
\hline Pause Time & 0 \\
\hline Speed & $5,10,15,20,25$ \\
\hline Mobility Model & Random Waypoint (RWM) \\
\hline Simulation Time & $100 \mathrm{Sec}$. \\
\hline
\end{tabular}

\section{B. Results and Discussions}

(i) Packet Delivery Ratio

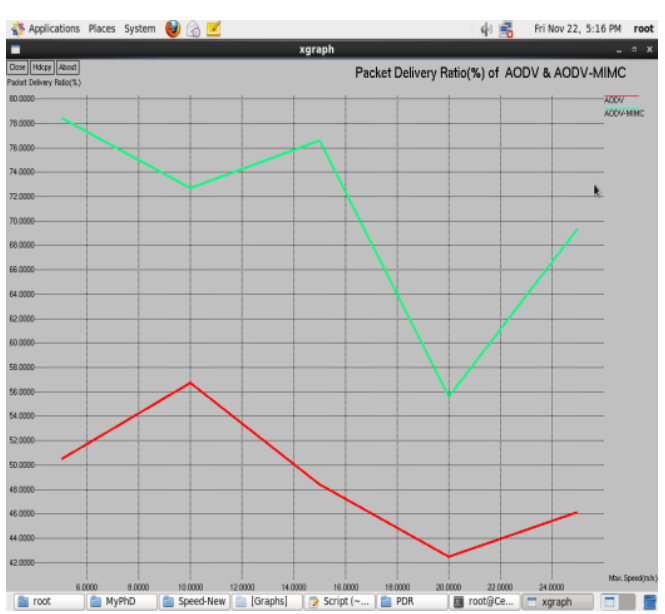

Fig 4: Packet Delivery Ratio (\%)

We observed from Fig. 4 that the Packet Delivery Ratio of AODV-MIMC routing protocol is remarkably improved than the Packet Delivery Ratio of AODV routing protocol.

\section{(ii) Throughput}

We observed from Fig.5 that the Throughput of AODV-MIMC routing protocol is also remarkably improved than the Throughput of AODV routing protocol

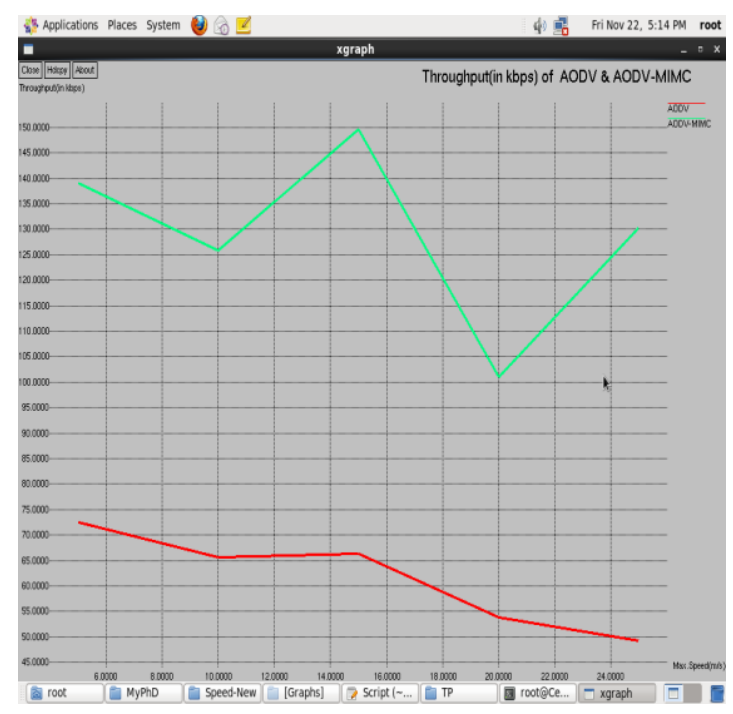

Fig 5: Throughput (in Kbps) 
(iii) Routing Overhead

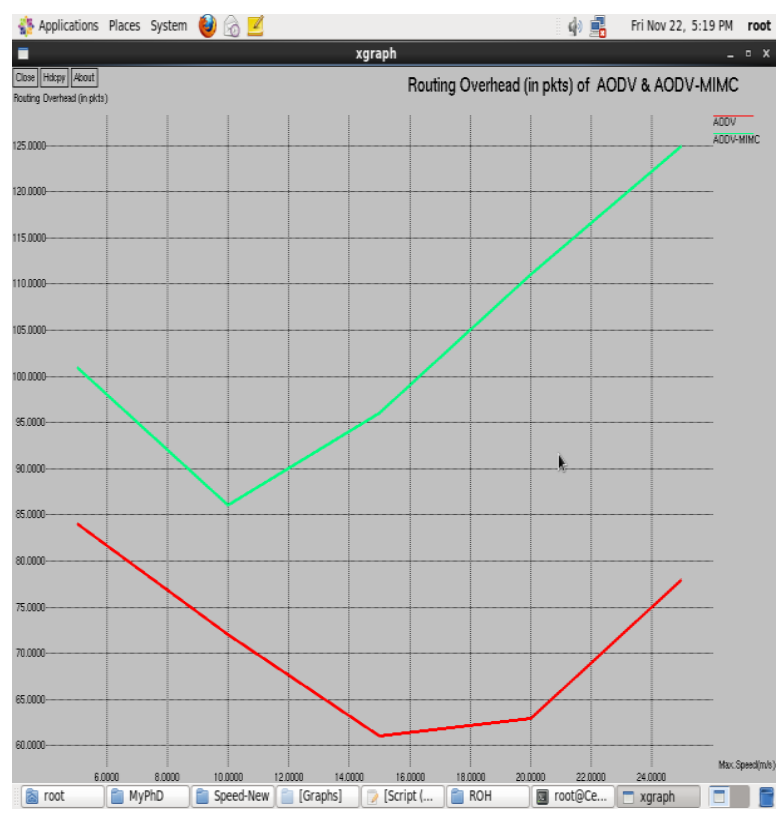

Fig 6: Routing Overhead (in Pkts)

When we compare the Routing Overhead of AODVMIMC routing protocol with the Routing Overhead of AODV routing protocol, the previous one is too high than the next one as shown in Fig.6.

\section{(iv) Normalized Routing Overhead}

When we compare the Normalized Routing Overhead of AODV-MIMC routing protocol with the Normalized Routing Overhead of AODV routing protocol, the previous one is too high than the next one as shown in Fig.7.

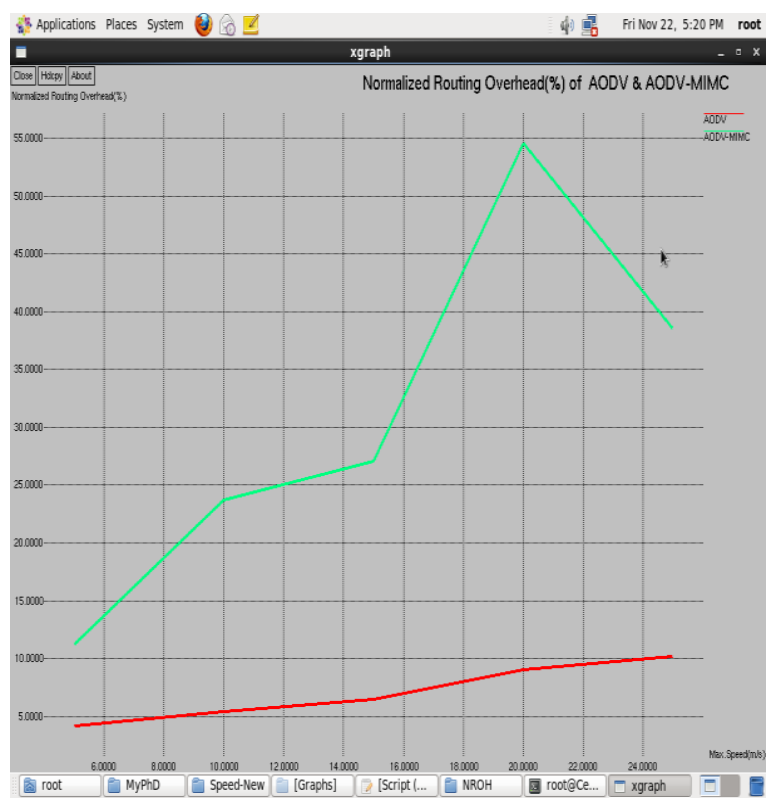

Fig 7: Normalized Routing Overhead (\%)

\section{(v) Average End-to-End Delay}

When we compare the average end-to-end delay of AODV-MIMC routing protocol with average end-toend delay of the AODV routing protocol, the previous one is too high than the next one as shown in Fig.8.

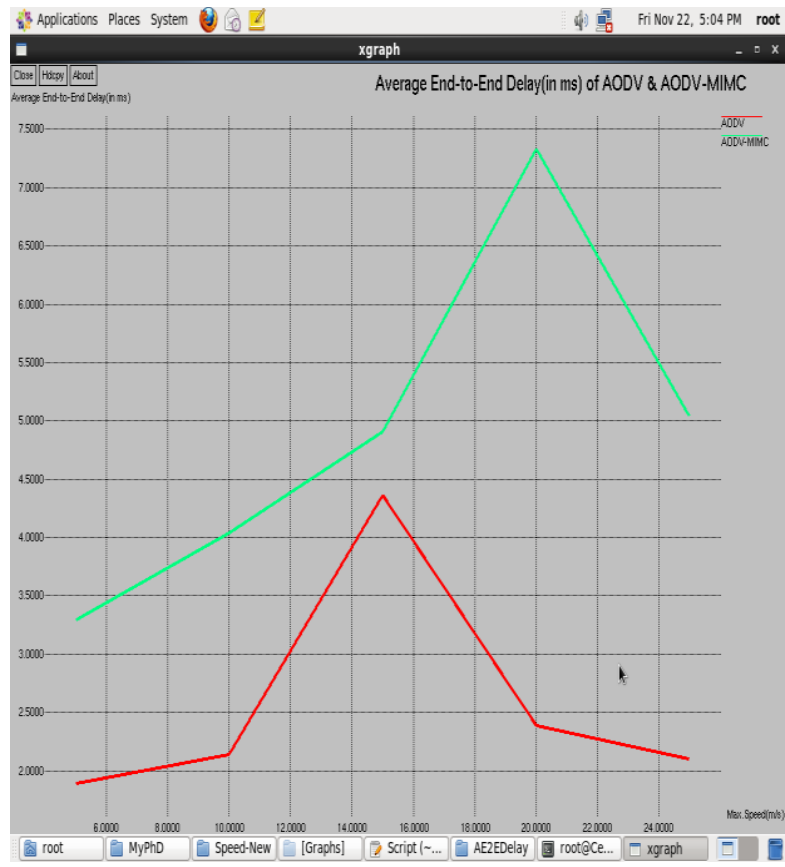

Fig 8: Average end-to-end delay (in ms)

\section{(vi) Packet Loss Ratio}

When we compare the Packet Loss Ratio of AODVMIMC routing protocol with the Packet Loss Ratio of AODV routing protocol, the previous one is too low than the next one as shown in Fig.9.

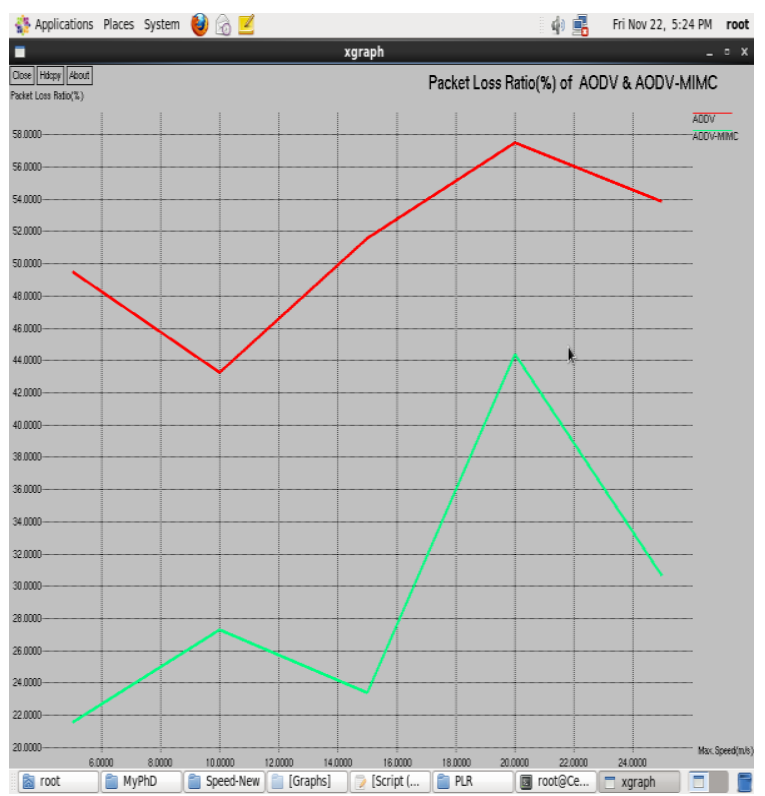

Fig 9: Packet Loss Ratio (\%) 
(vii) Total Energy consumed by nodes

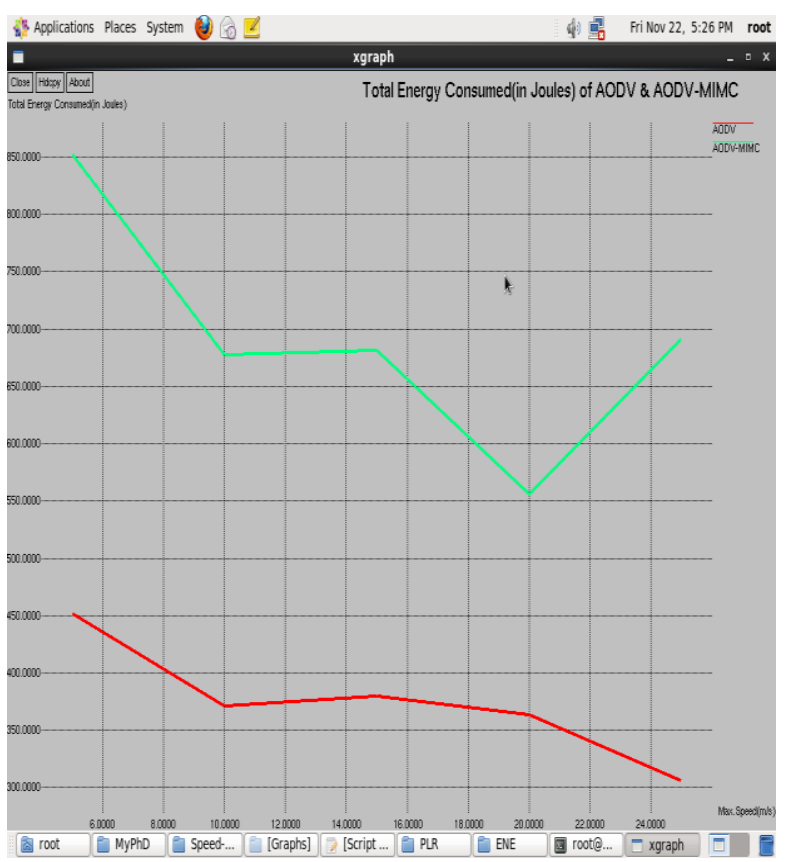

Fig 10: Total Energy consumed by nodes

It was found that the total energy consumed by nodes in AODV-MIMC routing protocol is too high than the total energy consumed by nodes in AODV routing protocol as shown in Fig.10.

(viii) Number of nodes dying

It was found that the number of nodes dying in AODV-MIMC routing protocol is low than the number of nodes dying in AODV routing protocol as shown in Fig.11.

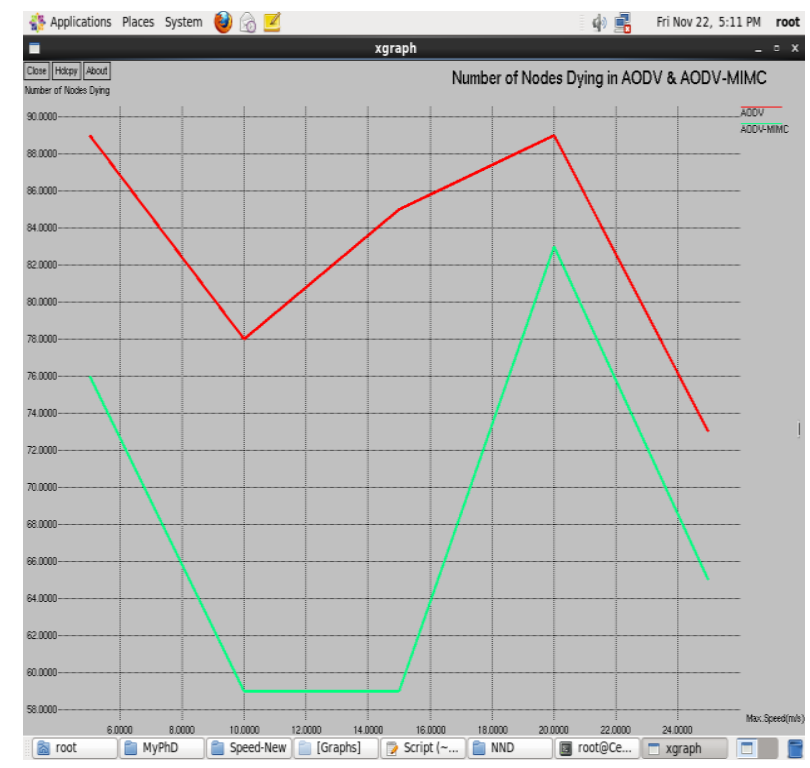

Fig 11: Number of nodes dying

(ix) Network Lifetime

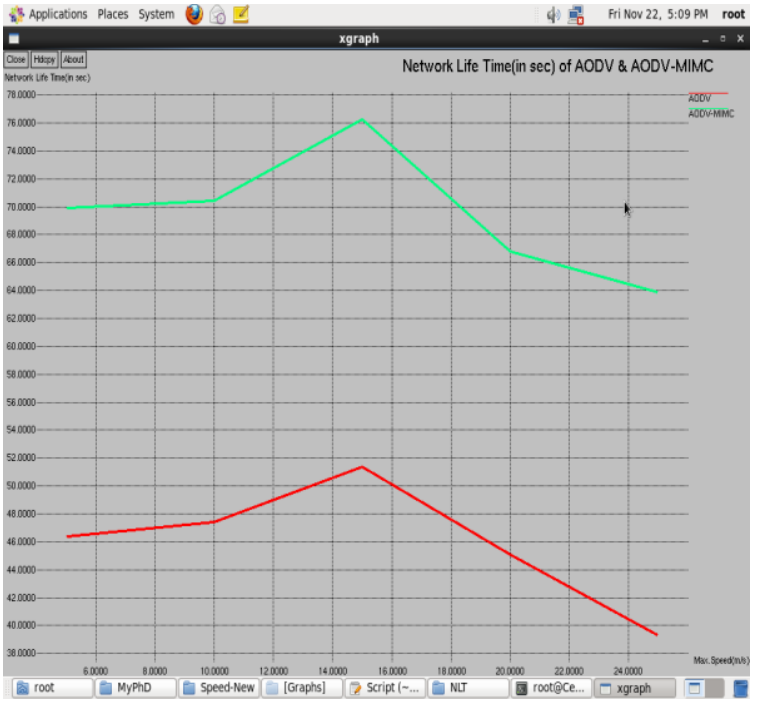

Figure 12 - Network Lifetime

It was found that the network lifetime of AODVMIMC routing protocol is much more improved than the network lifetime of AODV routing protocol as shown in Fig.12.

\section{CONCLUSIONS AND FUTURE WORK}

The Packet Delivery Ratio, Network Lifetime and Throughput of AODV-MIMC routing protocol is very much improved than the Packet Delivery Ratio, Network Lifetime and Throughput of AODV routing protocol. The Packet Loss Ratio of AODV-MIMC routing protocol is too low than the Packet Loss Ratio of AODV routing protocol. Total energy consumed by nodes, Average end-to-end delay and Normalized routing overhead of AODV-MIMC routing protocol is too high than the Total energy consumed by nodes, Average end-to-end delay and Normalized routing overhead of AODV routing protocol.

In future, we will concentrate on the reduction of either the average end-to-end delay or the total energy consumed by nodes or both in AODV-MIMC routing protocol because its performance is outstanding than the performance of AODV routing protocol.

\section{REFERENCES}

[1] Elizabeth M. Royer, C-K Toh, "A Review of Current Routing Protocols for Ad-Hoc Mobile Wireless Networks", IEEE Personal Communications, April 1999, pp.46-55.

[2] Mehran Abolhasan, Tadeusz Wysocki, and Eryk Dutkiewicz, "A review of routing protocols for mobile ad hoc networks", Ad Hoc Networks, June 2003, pp.1-22.

[3] S R. A. Calvo, J. P. Campo. "Adding Multiple Interface Support in NS-2", University of Cantabria, Jan. 2007, http://telecom.inescporto.pt/ rcampos/ucMultiIfacesSuppo rt.pdf.

[4] Trung-Tuan Luong, Bu-Sung Lee and C. K. Yeo. "Channel Allocation for Multiple Channels Multiple Interfaces Communication in Wireless Ad Hoc Networks". Lecture 
Notes in Computer Science, 2008, Volume 4982, NETWORKING 2008 Ad Hoc and Sensor Networks, Wireless Networks, Next Generation Internet, pp: 87-98.

[5] A. Raniwala, K. Gopalan, and T. Chiueh, "Centralized Channel Assignment and Routing Algorithms for MultiChannel Wireless Mesh Networks," Mobile Computing and Communications Review (MC2R) 2004, vol. 8, no. 2, pp. 50-65, Apr. 2004.

[6] P. Kyasanur and N. H. Vaidya, "Routing and Interface Assignment in Multi-Channel Multi-Interface Wireless Networks," in Proc. of IEEE WCNC 2005, vol. 4, pp. 2051-2056.

[7] H. Nguyen, U. Nguyen, "Channel assignment for multicast in multi-channel multi-radio wireless mesh networks," Wireless Communications and Mobile Computing, Published Online, Oct. 2008.

[8] C. Chereddi. Pradeep Kyasanur, Jungmin So and N. H. Vaidya. Multi-Channel Mesh Networks: Challenges and Protocols. IEEE Wireless Communications, April 2006.

[9] G. Zeng, B. Wang, et al., "Multicast Algorithms for MultiChannel Wireless Mesh Networks," IEEE ICNP, Oct. 2007.

[10] P. Kyasanur and N. Vaidya. Routing and Link-layer Protocols for Multi-Channel Multi-Interface Ad Hoc Wireless Networks. ACM SIGMOBILE MC2R, 10(1):3143, Jan 2006.

[11] Avallone, S., \& Akyildiz, I. F. (2008). A channel assignment algorithm for multi-radio wireless mesh networks. Computer Communications, 31(7), 1343-1353.

[12] Shin, M., Lee, S., \& Kim, Y. (2006). Distributed channel assignment for multi-radio wireless networks. In 2006 IEEE International Conference on Mobile Ad Hoc and Sensor Systems pp. 417-426.

[13] S. Das, C. Perkins and E. Royer, "Ad Hoc On Demand Distance Vector (AODV) Routing", IETF RFC3561, July 2003.

[14] C.P.Agrawal, O.P.Vyas, M.K.Tiwari, "Evaluation of varying mobility models \& network loads on DSDV protocol of MANETs", International Journal of Computer Science and Engineering,Vol. 1(2), pp. 40-46, 2009.

[15] P.Periyasamy and E.Karthikeyan, "Performance Evaluation of AOMDV Protocol based on various Scenario and Traffic Patterns", International Journal of Computer Science, Engineering and Applications (IJCSEA), Vol.1, No.6, 2011, pp. 33-48.

[16] Yumei Liu, Lili Guo, Huizhu Ma, Tao Jiang,"Energy efficient on demand multipath routing protocol for multihop ad hoc networks", ISSSTA-08, IEEE 10th International symposium on Spread spectrum and applications. Bologna, Italy, August 25-27 2008, pp-592597.
[17] Vikas Singla, Parveen Kakkar, "Traffic Pattern based performance comparison of Reactive and Proactive protocols of Mobile Ad-hoc Networks", International Journal of Computer Applications, Vol. 5(10), pp.16-20, 2010.

[18] Radhika Ranjan Roy, "Handbook of Mobile Ad Hoc Networks for Mobility Models", Springer, 2011.

[19] "The Network Simulator: ns-2". [Online]. Available: http://www.isi.edu/nsnam/ns/. [Accessed: 14-Nov-2012].

[20] Kevin Fall, K. Varadhan, "The ns Manual", University of Southern California, Information Sciences Institute (ISI). [Online]. Available: http://www.isi.edu/nsnam/ns/nsdocumentation.html. [Accessed: 14-Nov-2012].

[21] "NS-2 with Wireless and Mobility Extensions". [Online]. Available: http://www.monarch.cs.cmu.edu. [Accessed: 14-Nov-2012].

\section{AUTHORS BIOGRAPHY}

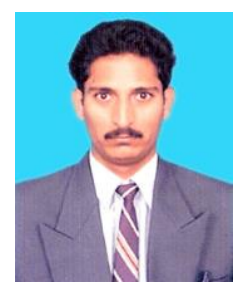

Periyasamy is working as an Assistant Professor in the Department of MCA, Sree Saraswathi Thyagaraja College, Pollachi, India. $\mathrm{He}$ is guiding students towards M.Phil. programme. He has published 5 papers in International Journals and also attended many conferences in both national and international levels and his area of interest is Mobile Ad hoc Networks Routing Protocols Design and Development. He is now pursuing Ph.D. from Bharathiar University, Coimbatore.

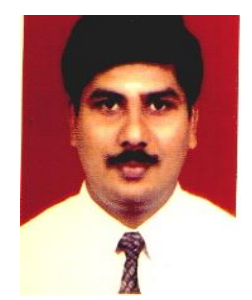

Dr.E.Karthikeyan born in 1974 at Dharapuuram completed PG degree in the year 1996 and Ph.D from Gandhigram University, Dindigul, India in 2008. He is guiding students towards Ph.D. programme and his area of research is Network Security and Cryptography and Advanced Networking. He has published 12 papers in International Journals and more than 15 conferences National and International level. He has also published a book entitled "Text Book on C: Fundamentals, Data structures and Programming" by PHI. He delivered lectures and conducted workshops in various colleges. He is a life member of CSI, CRSI, IASCT etc. He is a Editor-in-Chief for an International Journal of Advanced Networking and Applications.

How to cite this paper: P.Periyasamy, Dr.E.Karthikeyan,"Comparative Performance Analysis of AODV and AODVMIMC Routing Protocols for Mobile Ad hoc Networks", IJCNIS, vol.6, no.6, pp.54-60, 2014. DOI: 10.5815/ijcnis.2014.06.08 